PROCEEDINGS OF THE

AMERICAN MATHEMATICAL SOCIETY

Volume 135, Number 1, January 2007, Pages 169-179

S 0002-9939(06)08469-3

Article electronically published on June 22, 2006

\title{
DISINTEGRATION OF PROJECTIVE MEASURES
}

\author{
DORIN ERVIN DUTKAY AND PALLE E. T. JORGENSEN
}

(Communicated by David R. Larson)

\begin{abstract}
In this paper, we study a class of quasi-invariant measures on paths generated by discrete dynamical systems. Our main result characterizes the subfamily of these measures which admit a certain disintegration. This is a disintegration with respect to a random walk Markov process which is indexed by the starting point of the paths. Our applications include wavelet constructions on Julia sets of rational maps on the Riemann sphere.
\end{abstract}

\section{INTRODUCTION}

We prove a general theorem about endomorphisms of measure spaces, but it is motivated by our desire to use wavelet-type time/scale-self similarity in operator theory, and more generally outside the traditional context of wavelet theory; see, e.g., BrJo02. Hence our present setup is chosen with these general applications in mind. What is typical is that the starting point is geometric, and that a Hilbert space is not given at the outset. The best choice of Hilbert space in turn is dictated by the applications at hand.

Since wavelet decompositions use a special case of a general disintegration process for measures via transition operators [Rue89, Wal01], we feel that it is of interest to offer a direct and self-contained statement and proof of a necessary and sufficient condition for disintegration.

In its general form, the subject of disintegration of measures has its roots in the pioneering work by David Ruelle [Rue89] on transfer operators. This viewpoint was continued by Peter Walters Wal01 in ergodic theory, and in operator algebras by a series of papers by Jean Renault and co-workers, on the use of groupoids in representation theory and in dynamics; see [Ren03, KuRe03, AnRe01, Ren98, Ren80]. While our present setup and proofs are self contained, in contrast the groupoid approach is realized via representations of a certain non-abelian algebra of operators (a $C^{*}$-algebra); see also BJO04. Hence the other approaches use representations of non-abelian algebras, which in turn entail more restrictive assumptions on the endomorphism and on the transfer operator which is the starting point of the analysis. Jean Renault's approach is via a groupoid $C^{*}$-algebra Ren80. So in this approach, the first step is from endomorphism to groupoid.

Received by the editors August 16, 2004 and, in revised form, July 29, 2005.

2000 Mathematics Subject Classification. Primary 42C40, 42A16, 42A65, 43A65, 46G15, 47D07, 60G18.

Key words and phrases. Measures, projective limits, transfer operator, martingale, fixed-point. This work was supported in part by NSF grant DMS 0457491. 
Our present approach is rather direct, starting with a given endomorphism of a measure space. Yet our result Theorem 3.4 below belongs to the same circle of ideas as that of a related disintgeration theorem of Renault et al.; see, e.g., KuRe03, Theorems 2.8 and 3.5]. However, turning to our present setup, there are new elements, e.g., path space measures: We begin with a construction of families of path space measures, and there is no natural groupoid which is represented on our path space $X_{\infty}$ (see section 3 below). So Renault's approach does not apply directly.

Our starting point is an endomorphism in a measure space $X$. One might add that it is then possible to get a corresponding representation of Renault's groupoid built on $X$, or rather Renault's groupoid $C^{*}$-algebra. With this, we note that our construction is then in fact a dilation of Renault's representation. Having representations opens up ways of doing decomposition and disintegration; see also [BJO04]. This viewpoint further opens up some intriguing possibilities, and applications, which however we leave to a future paper.

\section{ENDOMORPHISMS AND MEASURES}

Let $X$ be a compact Hausdorff space, and let $r: X \rightarrow X$ be a given finite-to-one continuous endomorphism, mapping $X$ onto itself.

As an example, $r=r(z)$ could be a rational mapping of the Riemann sphere. In that case, we take $X$ to be the corresponding Julia set, or $X$ could be the state-space of a subshift associated to a $0-1$ matrix.

Due to work of Brolin [Bro and Ruelle Rue89, it is known that, for these examples, the dynamical system $(X, r)$ carries a unique maximal entropy or minimal free energy measure $\mu$. Specifically, for each point $x$ in $X, \mu$ distributes the "mass" equally on the finite number of solutions $y$ to $r(y)=x$. The measure $\mu$ is obtained by iterating this over the paths of inverse images under $r$.

In earlier papers, BrJo05, DuJo04a, DuJo04b, we showed that this structure in fact admits a rich family of wavelet bases. This wavelet construction is on a Hilbert space which is different from, but analogous to, $L^{2}(\mathbb{R})$, and the more familiar case of multiwavelets on the real line.

These are the wavelets corresponding to scaling $x$ to $N x$ by a fixed integer $N, N \geq 2$. In that case, $X=\mathbb{T}$, the circle in $\mathbb{C}$, and $r(z)=z^{N}$. So even in the "classical" case, there is a "unitary dilation" from $L^{2}(X)$ to $L^{2}(\mathbb{R})$. In this construction, the Haar measure on $\mathbb{T}$ "turns" into the Lebesgue measure on $\mathbb{R}$. In our general construction, we pass from $(X, r, \mu)$ to a Hilbert space built on a system $\left(X_{\infty}, \hat{r}, \hat{\mu}\right)$. Just as in the familiar case of $L^{2}(\mathbb{R})$, we shall be concerned with the interplay between the initial space and the dilated one. Our consideration of quasi-invariant measures is motivated by wavelet filters in the more familiar case of $L^{2}(\mathbb{R})$ wavelet analysis. See for example Dau92] and BrJo02. We stress that it is the dilated Hilbert space which carries wavelet bases. These bases are in turn parametrized by a family of functions $V$ on $X$, or on $\mathbb{T}$ in the classical case.

Our work so far on examples shows that this viewpoint holds promise for a deeper understanding of the harmonic analysis of Julia sets, and of other iteration systems.

In these cases, the analogue of $L^{2}(\mathbb{R})$ involves quasi-invariant measures $\hat{\mu}$ on a space $X_{\infty}$, built from $X$ in a way that closely follows the analogue of passing 
from $\mathbb{T}$ to $\mathbb{R}$ in wavelet theory; see DuJo04a for details. But the induced measure $\hat{\mu}$ on $X_{\infty}$ is now a path-space measure. The analogue of the unitary action of translations by the integers $\mathbb{Z}$ on $L^{2}(\mathbb{R})$, or equivalently multiplication in the Fourier dual, will be described in general below. For the Julia examples, it is the operator of multiplication by $z$ on $L^{2}(X, \mu)$, i.e., it is a normal operator. We get a corresponding covariant system on $X_{\infty}$, where multiplication by $f(z)$ is unitarily equivalent to multiplication by the composition $f(r(z))$. But this is now dilated onto the Hilbert space $L^{2}\left(X_{\infty}\right)$, and our dilated Hilbert space is defined from our path-space measures $\hat{\mu}$.

Hence all the issues from wavelet analysis of the mid-1980s, especially the pyramid algorithms, have analogues in this wider context. In other work, we use this to construct multiresolutions on the Julia sets and on other iteration systems, and our $r$-scale multiresolutions appear to reveal interesting spectral theory for Julia sets. Also, it seems further to offer a promising interplay between geometry and spectrum. None of this is yet well understood.

The purpose of this paper is to give an interconnection between the measures $\mu_{0}$ on $X$ and a family of $V$-quasi-invariant measures $\hat{\mu}$ on $X_{\infty}$. We characterize those $V$-quasi-invariant measures $\hat{\mu}$ on $X_{\infty}$ which admit a path-space decomposition in terms of the initial measure $\mu_{0}$ at the start of the paths. This is useful because it gives the $V$-quasi-invariant measure a more concrete form; see DuJo04a.

We recall the definitions: If $\mathfrak{B}$ is a sigma-algebra on a set $X$ and $r: X \rightarrow X$ is a finite-to-one, onto and measurable map, we denote it by

$$
X_{\infty}(r):=\left\{\left(x_{0}, x_{1}, \ldots\right) \in \prod_{n \in \mathbb{N}_{0}} X \mid r\left(x_{n+1}\right)=x_{n}, \text { for all } n \in \mathbb{N}_{0}\right\} .
$$

We denote the projections by $\theta_{n}: X_{\infty}(r) \ni\left(x_{0}, x_{1}, \ldots\right) \mapsto x_{n} \in X$. The union of the pull-backs $\theta_{n}^{-1}(\mathfrak{B})$ generates a sigma-algebra $\mathfrak{B}_{\infty}$. The map $r$ extends to a measurable bijection $\hat{r}: X_{\infty}(r) \rightarrow X_{\infty}(r)$ defined by

$$
\hat{r}\left(x_{0}, x_{1}, \ldots\right)=\left(r\left(x_{0}\right), x_{0}, x_{1}, \ldots\right) .
$$

Let $V: X \rightarrow[0, \infty)$ be a measurable, bounded function. We say that a measure $\mu_{0}$ on $(X, \mathfrak{B})$ has the fixed-point property if

$$
\int_{X} V f \circ r d \mu_{0}=\int_{X} f d \mu_{0}, \quad\left(f \in L^{\infty}\left(X, \mu_{0}\right)\right) .
$$

We say that a measure $\hat{\mu}$ on $\left(X_{\infty}(r), \mathfrak{B}_{\infty}\right)$ is $V$-quasi-invariant if

$$
d(\hat{\mu} \circ \hat{r})=V \circ \theta_{0} d \hat{\mu} .
$$

We recall the result from DuJo04b.

Theorem 2.1. There exists a one-to-one correspondence between measures $\mu_{0}$ on $X$ with the fixed-point property and $V$-quasi-invariant measures $\hat{\mu}$ on $X_{\infty}(r)$, given by

$$
\mu_{0}=\hat{\mu} \circ \theta_{0}^{-1} .
$$

Our main result, Theorem 3.4 below, applies to a class of measures on some extended spaces $X_{\infty}$, generalized solenoids. Our setup is as follows: Start with $\left(X, r, V, \mu_{0}\right)$ where $X$ is a measure space with an endomorphism $r, V$ is a weight function as specified, and $\mu_{0}$ is a $V$-strongly invariant measure. In wavelet applications (see DuJo04b],$V$ may have the form $V=\left|m_{0}\right|^{2}$ where $m_{0}$ is a wavelet low-pass filter. In the dyadic case, $X=\mathbb{T}$ (the circle or the one-torus) and 
$r: z \mapsto z^{2}$. Under suitable conditions on the so-called filter function $m_{0}$, we show in DuJo04b] that $\left(X_{\infty}, \mu_{\infty}\right)$ is measure theoretically equivalent to the real line $(\mathbb{R}$, Lebesgue measure), and so, in this case, the dilated Hilbert space is merely the familiar $L^{2}(\mathbb{R})$ of classical wavelet theory Dau92. In DuJo04a, we show that the same idea applies to the case when the system $\left(X, r, V, \mu_{0}\right)$ is as follows: $X:=X(r)$ is the Julia set of some fixed rational mapping $r(z)$ of one complex variable. (We also denote by $r$ the restriction to $X$ of the rational mapping $r(z)$.) Using a theorem of Brolin Bro about measures on Julia sets, we further describe in DuJo04a] a class of weight functions $V$ and measures $\mu_{0}$ on $X$ which satisfy the $V$-fixed point property considered in Theorem 3.1. We also describe in DuJo04a how our dilation construction then provides wavelet bases in the dilation Hilbert space $L^{2}\left(X_{\infty}, \mu_{\infty}\right)$. However, in our work in DuJo04a, DuJo04b] some cases are left open. This limits our approach to dilation/wavelet constructions for dynamical systems. The reason is that at the time we lacked a general disintegration formula for the relevant measure spaces. It is the purpose of the present paper to remedy this, and to prove such a theorem, specifically Theorem 3.4 below. We now turn to the path-space measures which are used in our theorem.

The significance of our path-space measures $\left\{P_{x} \mid x \in X\right\}$ is that they support our disintegration from Theorem 3.4 below. This is further elaborated in DuJo04a, DuJo04b. In the wavelet case, starting with $V=\left|m_{0}\right|^{2}$, let $P_{x}$ be the path-space measures from Proposition 3.3. We gave conditions in DuJo04a which imply that for a.e. $x, P_{x}$ is supported on the union $U$ of certain finite orbits for $r$. We outline how this union $U$ is naturally in a bijective correspondence with a copy of the integers $\mathbb{Z}$. This turns out to be consistent with the natural way the group $\mathbb{Z}$ acts by translation in wavelet theory of the Hilbert space $L^{2}(\mathbb{R})$.

Starting with $V$ and the corresponding $P_{x}$, we then get an important function $h(x):=P_{x}(\mathbb{Z})$. It turns out to be a fundamental eigenfunction of wavelet theory. In fact the function $h$ is known BrJo02 to be a fundamental minimal eigenfunction for the wavelet transfer operator $R_{V}$ (also called a Ruelle-Perron-Frobenius operator), studied first by W. Lawton in Law90. The function $h$ determines a number of important properties of a wavelet basis; see, e.g., Dau92, Chapter 6] and BrJo02 for additional details on this point. We further show in DuJo04b that $R_{V} h=h$ holds, and further that $h$ is an infinite-dimensional generalization of the familiar Perron-Frobenius right-eigenvector. This entails a suitable renormalization so that the Perron-Frobenius eigenvalue is 1 . The measures $\mu_{0}$ from Theorem 3.1 satisfying our $V$-fixed point property may then be viewed as generalizations of Perron-Frobenius left-eigenvectors. See also [BrJo02] and [Rue89] for details on infinite-dimensional Perron-Frobenius theory.

\section{Disintegration}

Let $X$ be a non-empty set, $\mathfrak{B}$ a sigma-algebra of subsets of $X$, and $r: X \rightarrow X$ an onto, finite-to-one, and $\mathfrak{B}$-measurable map.

We will assume in addition that we can label measurably the branches of the inverse of $r$. By this, we mean that the following conditions are satisfied:

$$
\text { The map } \quad \mathfrak{c}: X \ni x \mapsto \# r^{-1}(x)<\infty \text { is measurable. }
$$

We denote

$$
A_{i}:=\left\{x \in X \mid \mathfrak{c}(x)=\# r^{-1}(x) \geq i\right\} \quad(i \in \mathbb{N}) .
$$


Equation (3.1) implies that the sets $A_{i}$ are measurable. Also they form a decreasing sequence and, since the map is finite to one,

$$
X=\bigcup_{i=1}^{\infty}\left(A_{i+1} \backslash A_{i}\right)
$$

Then, we assume that there exist measurable maps $\tau_{i}: A_{i} \rightarrow X, i \in\{1,2, \ldots\}$, such that

$$
\begin{gathered}
r^{-1}(x)=\left\{\tau_{1}(x), \ldots, \tau_{\mathfrak{c}(x)}(x)\right\} \quad(x \in X), \\
\tau_{i}\left(A_{i}\right) \in \mathfrak{B}, \text { for all } i \in\{1,2, \ldots\} .
\end{gathered}
$$

Thus $\tau_{1}(x), \ldots, \tau_{\mathfrak{c}(x)}(x)$ is a list without repetition of the "roots" of $x, r^{-1}(x)$.

From (3.2) we also obtain that

$$
\tau_{i}\left(A_{i}\right) \cap \tau_{j}\left(A_{j}\right)=\emptyset, \text { if } i \neq j,
$$

and

$$
\bigcup_{i=1}^{\infty} \tau_{i}\left(A_{i}\right)=X
$$

In the sequel, we will use the following notation: For a function $f: X \rightarrow \mathbb{C}$, we denote

$$
f \circ \tau_{i}(x):=\left\{\begin{array}{cl}
f\left(\tau_{i}(x)\right), & \text { if } \quad x \in A_{i}, \\
0, & \text { if } \quad x \in X \backslash A_{i} .
\end{array}\right.
$$

Theorem 3.1. Let $(X, \mathfrak{B})$ and $r: X \rightarrow X$ be as above. Let $V: X \rightarrow[0, \infty)$ be a bounded $\mathfrak{B}$-measurable map. For a measure $\mu_{0}$ on $(X, \mathfrak{B})$, the following affirmations are equivalent:

(i) The measure $\mu_{0}$ has the fixed-point property (3.8).

(ii) There exists a non-negative, $\mathfrak{B}$-measurable map $\Delta$ (depending on $V$ and $\mu_{0}$ ) such that

$$
\sum_{r(y)=x} \Delta(y)=1, \text { for } \mu_{0} \text {-a.e. } x \in X,
$$

and

$$
\int_{X} V f d \mu_{0}=\int_{X} \sum_{r(y)=x} \Delta(y) f(y) d \mu_{0}(x), \text { for all } f \in L^{\infty}\left(X, \mu_{0}\right) .
$$

Moreover, when the affirmations are true, $\Delta$ is unique up to $V d \mu_{0}$-measure zero.

Proof. We first check the implication (ii) $\Rightarrow$ (i). We have, for $f \in L^{\infty}\left(X, \mu_{0}\right)$,

$$
\begin{aligned}
\int_{X} V f \circ r d \mu_{0} & =\int_{X} \sum_{r(y)=x} \Delta(y) f(r(y)) d \mu_{0}(x) \\
& =\int_{X} f(x) \sum_{r(y)=x} \Delta(y) d \mu_{0}(x)=\int_{X} f d \mu_{0},
\end{aligned}
$$

and (2.1) follows.

For the implication (i) $\Rightarrow$ (ii) we will need a lemma. 
Lemma 3.2. Let $\mu_{0}$ be a measure on $(X, \mathfrak{B})$ that satisfies (2.1). Then, for each $f \in L^{1}\left(X, \mu_{0}\right)$ there exists a function which we denote by $R_{\mu_{0}}(V f) \in L^{1}\left(X, \mu_{0}\right)$ such that

$$
\int_{X} V f g \circ r d \mu_{0}=\int_{X} R_{\mu_{0}}(V f) g d \mu_{0} \quad\left(g \in L^{\infty}\left(X, \mu_{0}\right)\right) .
$$

The operator $f \mapsto R_{\mu_{0}}(V f)$ has the following properties:

$$
\begin{gathered}
R_{\mu_{0}}(V)\left(=R_{\mu_{0}}(V \cdot \mathbf{1})\right)=\mathbf{1}, \text { pointwise } \mu_{0} \text {-a.e., } \\
R_{\mu_{0}}(V f h \circ r)=R_{\mu_{0}}(V f) h \quad\left(f, h \in L^{\infty}\left(X, \mu_{0}\right),\right. \\
R_{\mu_{0}}(V f) \geq 0 \text { if } f \geq 0 .
\end{gathered}
$$

Proof of Lemma 3.2. The positive functional

$$
g \mapsto \int_{X} V f g \circ r d \mu_{0} \quad\left(g \in L^{\infty}\left(X, \mu_{0}\right)\right)
$$

defines a measure on $(X, \mathfrak{B})$ which is absolutely continuous with respect to $\mu_{0}$. Indeed, if $E \in \mathfrak{B}$ has $\mu_{0}(E)=0$, then by (2.1)

$$
\int_{X} V \chi_{E} \circ r d \mu_{0}=\int_{X} \chi_{E} d \mu_{0}=0,
$$

therefore $r^{-1}(E)$ has $V d \mu_{0}$-measure zero so

$$
\int_{X} V f \chi_{E} \circ r d \mu_{0}=0 .
$$

Denoting by $R_{\mu_{0}}(V f)$ the Radon-Nikodym derivative, the relation (3.8) is obtained.

Taking $f=\mathbf{1}$, we obtain, for all $g \in L^{\infty}\left(X, \mu_{0}\right)$,

$$
\int_{X} R_{\mu_{0}}(V) g d \mu_{0}=\int_{X} V g \circ r d \mu_{0}=\int_{X} g d \mu_{0},
$$

where we used (2.1) in the last step. Thus (3.9) follows.

Next, for all $g \in L^{\infty}\left(X, \mu_{0}\right)$ we have

$$
\int_{X} R_{\mu_{0}}(V f h \circ r) g d \mu_{0}=\int_{X} V f h \circ r g \circ r d \mu_{0}=\int_{X} R_{\mu_{0}}(V f) h g d \mu_{0},
$$

and this implies (3.10).

Now take $f \geq 0, f \in L^{1}\left(X, \mu_{0}\right)$. For all $g \in L^{\infty}\left(X, \mu_{0}\right)$ with $g \geq 0$, we have

$$
\int_{X} R_{\mu_{0}}(V f) g d \mu_{0}=\int_{X} V f g \circ r d \mu_{0} \geq 0
$$

and (3.11) follows.

We return to the proof of the theorem. We prove that there exist some nonnegative, measurable function $\Delta$ on $X$, such that

$$
\left(R_{\mu_{0}}(V f)\right)(x)=\sum_{i=1}^{\infty} \Delta\left(\tau_{i}(x)\right) f\left(\tau_{i}(x)\right), \text { for } \mu_{0} \text {-a.e. } x \in X \quad\left(f \in L^{1}\left(X, \mu_{0}\right)\right) .
$$

Note that every $f \in L^{1}\left(X, \mu_{0}\right)$ can be written as

$$
f=\sum_{i=1}^{N}\left(f \circ \tau_{i}\right) \circ r \chi_{\tau_{i}\left(A_{i}\right)} .
$$


Indeed, if $x \in X$, then, by (3.5), $x \in \tau_{i}\left(A_{i}\right)$ for some $i \in\{1,2, \ldots\}$, and

$$
x \in r^{-1}(r(x))=\left\{\tau_{1}(r(x)), \ldots, \tau_{\mathfrak{c}(r(x))}(r(x))\right\} .
$$

Therefore $x=\tau_{i}(r(x))$ and (3.13) follows. Note also that, for all $x \in X$, the sum in (3.13) is actually finite. It has at most $\mathfrak{c}(x)$ terms.

The function $f \circ \tau_{i}$ is measurable because $\tau_{i}$ is.

Applying $R_{\mu_{0}}$ and using (3.10) we obtain that

$$
R_{\mu_{0}}(V f)=\sum_{i=1}^{\infty} R_{\mu_{0}}\left(V \chi_{\tau_{i}\left(A_{i}\right)}\right) f \circ \tau_{i}
$$

A little argument is needed for this, to deal with the infinite sum. If $S_{n}$ are the partial sums $S_{n}:=\sum_{i=1}^{n} \chi_{\tau_{i}\left(A_{i}\right)} f \circ \tau_{i} \circ r$, then they converge pointwise and dominate to the limit $f$. The definition of $R_{\mu_{0}}$ shows that $R_{\mu_{0}}\left(V S_{n}\right)$ converges weakly to $R_{\mu_{0}}(V f)$. However, the sum in $R_{\mu_{0}}(V f)$ is pointwise finite, because any $x \in X$ belongs to only finitely many $A_{i}$ 's, so $f \circ \tau_{i}(x)=0$, except for finitely many $i$ 's. Hence (3.14) holds.

Then define

$$
\Delta\left(\tau_{i}(x)\right)=\left(R_{\mu_{0}}\left(V \chi_{\tau_{i}\left(A_{i}\right)}\right)\right)(x) \quad\left(x \in A_{i}\right) .
$$

Since $\left(\tau_{i}(X)\right)_{i=1, \infty}$ form a partition of $X$, (3.15) defines the function $\Delta$ on $X$.

We claim that $\Delta$ is measurable. Take $E$ to be a Borel subset of $\mathbb{R}$. Then

$$
\begin{aligned}
\Delta^{-1}(E) & =\bigcup_{i=1}^{\infty}\left(\Delta^{-1}(E) \cap \tau_{i}\left(A_{i}\right)\right) \\
& =\bigcup_{i=1}^{\infty}\left(\tau_{i}\left(\left(\Delta \circ \tau_{i}\right)_{\left.\right|_{A_{i}}}^{-1}(E)\right)\right) \\
& =\bigcup_{i=1}^{\infty}\left(\tau_{i}\left(\left(R_{\mu_{0}}\left(V \chi_{\tau_{i}\left(A_{i}\right)}\right)\right)^{-1}(E)\right)\right) \\
& =\bigcup_{i=1}^{\infty}\left(r^{-1}\left(\left(R_{\mu_{0}}\left(V \chi_{\tau_{i}\left(A_{i}\right)}\right)\right)^{-1}(E)\right) \cap \tau_{i}\left(A_{i}\right)\right),
\end{aligned}
$$

and since the functions $R_{\mu_{0}}\left(V \chi_{\tau_{i}\left(A_{i}\right)}\right)$ are measurable, it follows that $\Delta$ is too.

Now the equations (3.14) and (3.15) imply (3.12). Plugging (3.12) into (3.8) we obtain (3.7).

$$
\sum_{i=1}^{\infty} \Delta \circ \tau_{i}=R_{\mu_{0}}(V \mathbf{1})=1
$$

where we used (3.9) in the last step. This proves (3.6).

To prove the uniqueness, let $\Delta^{\prime}$ be another such function. Considering in (3.7) functions $f$ supported on $\chi_{\tau_{i}(X)}$, we obtain that

$$
\Delta\left(\tau_{i}(x)\right)=\Delta^{\prime}\left(\tau_{i}(x)\right), \text { for } \mu_{0} \text {-a.e. } x \in A_{i} .
$$


But

$$
\begin{aligned}
\left\{x \in X \mid \Delta(x) \neq \Delta^{\prime}(x)\right\} & =\bigcup_{i=1}^{\infty}\left\{x \in \tau_{i}\left(A_{i}\right) \mid \Delta\left(\tau_{i}(r(x))\right) \neq \Delta^{\prime}\left(\tau_{i}(r(x))\right)\right\} \\
& =\bigcup_{i=1}^{\infty}\left(\tau_{i}\left(A_{i}\right) \cap r^{-1}\left(\left\{y \in X, \mid \Delta\left(\tau_{i}(y)\right) \neq \Delta^{\prime}\left(\tau_{i}(y)\right)\right\}\right)\right) .
\end{aligned}
$$

However, the argument at the beginning of the proof shows that if a set $E$ has $\mu_{0}(E)=0$, then $r^{-1}(E)$ has $V d \mu_{0}$-measure 0 . Thus each term of the union has $V d \mu_{0}$-measure 0 , and the theorem is proved.

3.1. Path measures. Each point in $\left(x_{0}, x_{1}, \ldots\right) \in X_{\infty}(r)$ is determined by a point $x_{0} \in X$ and a choice of a root $x_{1} \in r^{-1}\left(x_{0}\right)$, a root $x_{2} \in r^{-1}\left(x_{1}\right) \subset r^{-2}\left(x_{0}\right)$, and so on.

If the point $x_{0}$ is fixed, then to specify a point in $X_{\infty}(r)$ is to specify the choice of roots, or the choice of a path

$$
x_{0} \stackrel{r}{\longleftarrow} x_{1} \stackrel{r}{\longleftarrow} \ldots \stackrel{r}{\longleftarrow} x_{n} \stackrel{r}{\longleftarrow} x_{n+1} \stackrel{r}{\longleftarrow} \ldots
$$

We will denote the set of these paths by

$$
\Omega_{x_{0}}:=\left\{\left(x_{1}, x_{2}, \ldots\right) \mid\left(x_{0}, x_{1}, x_{2}, \ldots\right) \in X_{\infty}(r)\right\} .
$$

The set can be regarded as a subset of $X_{\infty}(r)$, namely the subset of points in $X_{\infty}(r)$ that have the first coordinate equal to $x_{0}$. It can also be regarded as the projective limit of the following diagram:

$$
r^{-1}\left(x_{0}\right) \stackrel{r}{\longleftarrow} r^{-2}\left(x_{0}\right) \stackrel{r}{\longleftarrow} \ldots \stackrel{r}{\longleftarrow} r^{-n}\left(x_{0}\right) \stackrel{r}{\longleftarrow} r^{-(n+1)}\left(x_{0}\right) \stackrel{r}{\longleftarrow} \ldots
$$

We can construct a Radon measure $P_{x_{0}}$ on $\Omega_{x_{0}}$ using $\Delta$ to assign probabilities to the choices of roots.

Proposition 3.3. Let $(X, \mathfrak{B})$ and $r: X \rightarrow X$ and be as above, and let $D: X \rightarrow$ $[0, \infty)$ be a measurable function with the property that

$$
\sum_{r(y)=x} D(y)=1
$$

Denote

$$
D^{(n)}:=D \cdot D \circ r \cdot \ldots \cdot D \circ r^{n-1} \quad(n \in \mathbb{N}), \quad D^{(0)}:=1 .
$$

Then for each $x_{0} \in X$, there exists a Radon probability measure $P_{x_{0}}$ on $\Omega_{x_{0}}$ such that, if $f$ is a bounded measurable function on $\Omega_{x_{0}}$ which depends only on the first $n$ coordinates $x_{1}, \ldots, x_{n}$, then

$$
\int_{\Omega_{x_{0}}} f(\omega) d P_{x_{0}}(\omega)=\sum_{r^{n}\left(x_{n}\right)=x_{0}} D^{(n)}\left(x_{n}\right) f\left(x_{1}, \ldots, x_{n}\right) .
$$

Proof. To check the consistency, take a measurable function $f$ which depends only on the first $n$ coordinates $x_{1}, \ldots, x_{n}$. We regard it as a function which depends on the first $n+1$ coordinates and check that the two formulas in (3.18) (one for $n$, one 
for $n+1)$ give the same result:

$$
\begin{aligned}
\int_{\Omega_{x_{0}}} f(\omega) d P_{x_{0}}(\omega) & =\sum_{r^{n+1}\left(x_{n+1}\right)=x_{0}} D^{(n+1)}\left(x_{n+1}\right) f\left(x_{1}, \ldots, x_{n+1}\right) \\
& =\sum_{r^{n}\left(x_{n}\right)=x_{0}} f\left(x_{1}, \ldots, x_{n}\right) D^{(n)}\left(x_{n}\right) \sum_{r\left(x_{n+1}\right)=x_{n}} D\left(x_{n+1}\right) \\
& =\sum_{r^{n}\left(x_{n}\right)=x_{0}} D^{(n)}\left(x_{n}\right) f\left(x_{1}, \ldots, x_{n}\right),
\end{aligned}
$$

so $P_{x_{0}}$ is well defined. Since the functions which depend only on finitely many coordinates form a dense subalgebra of bounded functions (according to the StoneWeierstrass theorem), we can use Riesz' theorem to obtain the probability measure $P_{x_{0}}$ on $\Omega_{x_{0}}$. The application of Stone-Weierstrass is justified because when the point $x$ is fixed, we may use Tychonoff-compactness of the usual infinite product $\Omega:=\mathbb{Z}_{N} \times \mathbb{Z}_{N} \times \ldots$. But since we may have a variable number of preimages, we must first construct a dense subalgebra of bounded functions, and then the measures $\left(P_{x}\right)$ by using the structure of the measure space $X_{\infty}(r)$ defined above.

3.2. Main theorem. The next theorem shows that the $V$-quasi-invariant measure $\hat{\mu}$ on $X_{\infty}(r)$ associated to $\mu_{0}$ can be disintegrated through the measures $P_{x_{0}}$ associated to the function $\Delta$.

Theorem 3.4. Let $(X, \mathfrak{B})$. $r: X \rightarrow X$ and $V: X \rightarrow[0, \infty)$ be as above. Let $\mu_{0}$ be a measure on $(X, \mathfrak{B})$ with the fixed point property (2.1). Let $\Delta: X \rightarrow[0,1]$ be the function associated to $V$ and $\mu_{0}$ as in Theorem [3.1, and let $\hat{\mu}$ be the unique $V$-quasi-invariant measure on $X_{\infty}(r)$ with

$$
\hat{\mu} \circ \theta_{0}^{-1}=\mu_{0}
$$

as in Theorem 2.1. For $\Delta$, we define the measures $P_{x_{0}}$ as in Proposition 3.3. Then, for all bounded measurable functions $f$ on $X_{\infty}(r)$,

$$
\int_{X_{\infty}(r)} f d \hat{\mu}=\int_{X} \int_{\Omega_{x_{0}}} f\left(x_{0}, \omega\right) d P_{x_{0}}(\omega) d \mu_{0}\left(x_{0}\right) .
$$

Proof. By density, it is enough to prove the theorem for functions $f$ on $X_{\infty}(r)$ which depend only on finitely many coordinates. So, assume $f=g \circ \theta_{n}$, with $g$ bounded and $\mathfrak{B}$ measurable. Then $g \circ \theta_{n}$ will depend only on $x_{0}$ and $x_{1}, \ldots, x_{n}$,

$$
g \circ \theta_{n}\left(x_{0}, x_{1}, \ldots, x_{n}\right)=g\left(x_{n}\right) .
$$

From DuJo04b, Theorem 3.3], we recall that if $\mu_{n}:=\hat{\mu} \circ \theta_{n}^{-1}$, then $d \mu_{n}=$ $V^{(n)} d \mu_{0}$. Thus

$$
\int_{X_{\infty}(r)} g \circ \theta_{n} d \hat{\mu}=\int_{X} g d \mu_{n}=\int_{X} V^{(n)} g d \mu_{0} .
$$

Applying Lemma 3.2, we obtain by induction that

$$
\begin{gathered}
\int_{X} V^{(n)} g d \mu_{0}=\int_{X} V g V^{(n-1)} \circ r d \mu_{0}=\int_{X} R_{\mu_{0}}(V g) V^{(n-1)} d \mu_{0} \\
=\ldots=\int_{X} R_{\mu_{0}}^{n}(V g) d \mu_{0} .
\end{gathered}
$$


Also, using induction on (3.12), we obtain that

$$
\begin{gathered}
\left(R_{\mu_{0}}^{n}(V g)\right)\left(x_{0}\right)=\sum_{r^{n}\left(x_{n}\right)=x_{0}} \Delta^{(n)}\left(x_{n}\right) g\left(x_{n}\right) \\
=\int_{\Omega_{x_{0}}}\left(g \circ \theta_{n}\right)\left(x_{0}, \omega\right) d P_{x_{0}}(\omega) .
\end{gathered}
$$

Combining these equalities we obtain (3.19).

\section{ACKNOWLEDGEMENTS}

We are pleased to thank an anonymous referee for calling our attention to the connection to groupoids, and in particular to the work of Jean Renault et al., stressing that this is an important alternative approach to disintegration based on transfer operators. In addition, both authors acknowledge encouragement and helpful discussions with David Larson, and partial support from the National Science Foundation, DMS 0139473, and DMS 0457581.

\section{REFERENCES}

[AnRe01] Claire Anantharaman; Jean Renault, Amenable groupoids. Groupoids in analysis, geometry, and physics (Boulder, CO, 1999), 35-46, Contemp. Math., 282, Amer. Math. Soc., Providence, RI, 2001. MR1855241(2002g:46110)

[BJO04] Ola Bratteli; Palle E. T. Jorgensen; Vasyl Ostrovskyi, Representation theory and numerical AF-invariants. The representations and centralizers of certain states on $\mathcal{O}_{d}$, Mem. Amer. Math. Soc. 168 (2004), no. 797, xviii+178 pp. MR2030387 (2005i:46069)

[BrJo02] O. Bratteli; P.E.T. Jorgensen, Wavelets through a looking glass: The world of the spectrum, Appl. Numerical and Harmonic Anal., Birkhauser, Boston, 2002. MR1913212 (2003i:42001)

[BrJo05] Ola Bratteli; Palle E. T. Jorgensen, Preprint in preparation.

[Bro] H. Brolin, Invariant sets under iteration of rational functions, Arkiv för Matematik, 6, 1965, 103-144. MR0194595 (33:2805)

[Dau92] I. Daubechies, Ten lectures on wavelets, CBMS-NSF, Regional Conf. Ser. SIAM, vol. 61, 1992. MR 1162107 (93e:42045)

[DuJo04a] D.E. Dutkay; P.E.T. Jorgensen, Martingales, endomorphisms, and covariant systems of operators in Hilbert space, preprint 2004, arxiv math.CA/0407330

[DuJo04b] D.E. Dutkay; P.E.T. Jorgensen, Hilbert spaces of martingales supporting certain substitution-dynamical systems. Conform. Geom. Dyn. 9 (2005), 24-45. MR2133804 (2006a:37005)

[FLM] A. Freire; A. Lopes; R. Mane, An invariant measure for rational maps, Bol. Soc. Brasil. Mat. 14 (1983), 45-62. MR0736568 (85m:58110b)

[KuRe03] Alex Kumjian; Jean Renault, KMS states on $C^{*}$-algebras associated to expansive maps, Preprint: arXiv/math.OA/0305044.

[Law90] Wayne M. Lawton, Tight frames of compactly supported affine wavelets, J. Math. Phys. 31 (1990), 1898-1901. MR1067632 (92a:81068)

[Mane] R. Mane, On the uniqueness of the maximizing measure for rational maps, Bol. Soc. Bras. Mat. 14, 1983, 27-43. MR0736567 (85m:58110a)

[Ren80] Jean Renault, A groupoid approach to $C^{*}$-algebras, Lecture Notes in Mathematics, 793. Springer, Berlin, 1980. ii+160 pp. MR0584266 (82h:46075)

[Ren98] Jean Renault, Cuntz-like algebras, Operator theoretical methods (Timişoara, 1998), 371-386, Theta Found., Bucharest, 2000. MR.1770333 (2001g:46130)

[Ren03] Jean Renault, AF equivalence relations and their cocycles, Operator algebras and mathematical physics (Constanţa, 2001), 365-377, Theta, Bucharest, 2003. MR2018241 (2005e:46129) 
[Rue89] D. Ruelle, The thermodynamic formalism for expansive maps, Comm. Math. Phys. 125 (1989), 239-262. MR1016871 (91a:58149)

[Wal01] Peter Walters, Convergence of the Ruelle operator for a function satisfying Bowen's condition, Trans. Amer. Math. Soc. 353 (2001), 327-347. MR.1783787(2001g:37029)

Department of Mathematics, Hill Center-Busch Campus, Rutgers, The State UniverSity of New Jersey, 110 Frelinghuysen Road, Piscataway, New Jersey 08854-8019

E-mail address: ddutkay@math.rutgers.edu

Department of Mathematics, The University of Iowa, 14 Maclean Hall, Iowa City, IowA 52242-1419

E-mail address: jorgen@math.uiowa.edu 